\title{
ON SPECIAL PIECES, THE SPRINGER CORRESPONDENCE, AND UNIPOTENT CHARACTERS
}

\author{
PRAMOD N. ACHAR AND DANIEL S. SAGE
}

\begin{abstract}
Let $G$ be a reductive algebraic group over the algebraic closure of a finite field $\mathcal{F}_{q}$ of good characteristic. In this paper, we demonstrate a remarkable compatibility between the Springer correspondence for $G$ and the parametrization of unipotent characters of $G\left(\mathcal{F}_{q}\right)$. In particular, we show that in a suitable sense, "large" portions of these two assignments in fact coincide. This extends earlier work of Lusztig on Springer representations within special pieces of the unipotent variety.
\end{abstract}

\section{INTRODUCTION}

Let $G$ be a reductive algebraic group, and let $W$ be its Weyl group. The Springer correspondence, which we denote by $\nu$, assigns to each irreducible representation of $W$ an irreducible equivariant local system on a unipotent class of $G$. On the other hand, part of Lusztig's parametrization of unipotent characters of finite reductive groups is a map $\kappa$ assigning to each irreducible representation of $W$ an element of a certain finite set, whose members may be thought of as irreducible equivariant local systems on certain finite groups.

The main goal of this paper is demonstrate a certain compatibility between these two maps. Both maps assign to each representation of $W$ an orbit in some space and a representation of an isotropy group. Our main result asserts that if we restrict to our attention to a given two-sided cell (in a sense to be made precise later), then there is a natural order-preserving bijection between the two sets of orbits, and that the relevant isotropy groups are canonically isomorphic. Furthermore, we show that if we then identify corresponding orbits and isotropy groups, then the Springer correspondence and Lusztig's map $\kappa$ actually coincide.

This theorem generalizes various results due to Lusztig [8]. For exceptional groups, its proof amounts to poring over tables from [3]. We will indicate in Section 2 exactly what poring is to be done, but we will not tabulate the results here. (Some of the calculations required for the exceptional groups case were first carried out by Kottwitz.) The proof for classical groups is carried out in Sections 3 and 4 . In Section 3, we discuss various general phenomena in the classical groups, and produce an outline of a proof that is independent of type but, of course, lacking in combinatorial details. Section 4 supplies these details in full for type $C$ and somewhat more sparsely in types $B$ and $D$.

It has often been the case that "combinatorial coincidences" in representation theory hint at deep underlying relationships between superficially disparate phenomena, and one wonders whether that is the case here. Since the Springer correspondence is intimately related to the theory of character sheaves, which enjoy a classification similar to that of characters of finite reductive groups, perhaps there 
is a purely geometric proof of the results of this paper in the character sheaf setting. (A small hint about the geometry in the problem appears in Lusztig's paper [8]: he comments on the relationship between the main results of that paper and a theorem of Kraft-Procesi [4] on the geometry of special pieces in the classical groups.) Such a proof would certainly shed light on why the results of this paper are true; perhaps it would also shed light on other matters in the connections between character sheaves, unipotent characters, and two-sided cells.

We are grateful to Robert Kottwitz for several valuable discussions as well as for sharing his exceptional groups calculations with us.

\section{BACKGROUND AND DEFINITIONS}

2.1. Unipotent classes and Lusztig's canonical quotient group. Let $\mathcal{U}$ denote the unipotent variety in $G$. For any $G$-stable subvariety $Z$ of $\mathcal{U}$, let $\mathcal{O}(Z)$ be the set of unipotent classes contained in $Z$. For each class $C \in \mathcal{O}(\mathcal{U})$, we denote by $A(C)$ the component group of the centralizer of some point $u \in C$ : that is, $A(C)=G^{u} /\left(G^{u}\right)^{\circ}$. (Different choices of $u$ clearly result in isomorphic groups $A(C)$; moreover, the isomorphism is determined up to an inner automorphism.) Next, with $Z$ as above, let

$$
X(Z)=\{(C, \rho) \mid C \in \mathcal{O}(Z) \text { and } \rho \in \operatorname{Irr}(A(C))\} .
$$

Of course, this set can be identified with the set of irreducible $G$-equivariant local systems on $G$-orbits in $Z$.

In particular, we may regard the Springer correspondence as a map $\nu: \operatorname{Irr}(W) \rightarrow$ $X(\mathcal{U})$. If $(C, \rho)$ is in the image of $\nu$, we write $\chi_{C, \rho}$ for its preimage.

Next, for a given unipotent class $C$, let

$$
K=\bigcap_{\begin{array}{c}
\rho \in \operatorname{Irr}(A(C)) \\
\chi C, \rho \text { and } \chi C, 1 \text { are in the } \\
\text { same two-sided cell }
\end{array}} \operatorname{ker} \rho \text { and } \bar{A}(C)=A(C) / K .
$$

This group is called Lusztig's canonical quotient of $A(C)$. We further define

$$
X^{0}(Z)=\{(C, \rho) \mid C \in \mathcal{O}(Z) \text { and } \rho \in \operatorname{Irr}(\bar{A}(C))\} .
$$

This set can be identified with a subset of $X(Z)$, by pulling back representations of $\bar{A}(C)$ to $A(C)$.

2.2. Special pieces. A special piece is, by definition, the union of a special unipotent class $C_{0}$ and those unipotent classes $C$ in its closure with the property that for any other special class $C_{1} \subsetneq \bar{C}_{0}$, we have $C \not \subset \overline{C_{1}}$. By a result of Spaltenstein [10], the full unipotent variety is the disjoint union of the various special pieces. Evidently, there is a one-to-one correspondence between special pieces and two-sided cells of $W$ : given a two-sided cell $\mathbf{c}$, we denote by $P_{\mathbf{c}}$ the special piece determined by the special unipotent class $C_{0}$ with the property that $\chi_{C_{0}, 1}$ is the unique special character in $\mathbf{c}$.

Let $\operatorname{Irr}(W)_{\mathbf{c}}$ denote the set of irreducible characters of $W$ belonging to c. Lusztig has shown [8] that a given unipotent class $C$ is contained in $P_{\mathbf{c}}$ if and only if $\chi_{C, 1} \in \operatorname{Irr}(W)_{\mathbf{c}}$. Comparing this fact with the definition of $X^{0}(\mathcal{U})$, we observe that for any $\chi \in \operatorname{Irr}(W)_{\mathbf{c}}$, the following two conditions are equivalent:

- $\nu(\chi)=(C, \rho)$ where $C$ is contained in $P_{\mathbf{c}}$ (that is, $\nu$ sends $\chi$ to the "correct" special piece) 
- $\nu(\chi) \in X^{0}(\mathcal{U})$.

2.3. The parametrization of unipotent characters. Let $\mathbf{c}$ be a two-sided cell of $W$. In [6], Lusztig defined a certain finite group $\mathcal{G}_{\mathbf{c}}$ attached to $\mathbf{c}$. This group acts on itself by conjugation. Let $Z \subset \mathcal{G}_{\mathbf{c}}$ be a subset that is stable under this action. By analogy with the unipotent variety of $G$, we define $\mathcal{O}(Z)$ to be the set of $\mathcal{G}_{\mathbf{c}}$-orbits (that is, conjugacy classes) in $Z$, and for any $y \in \mathcal{O}\left(\mathcal{G}_{\mathbf{c}}\right)$, we define $I(y)$ to be the centralizer $\mathcal{G}_{\mathbf{c}}^{x} \subset \mathcal{G}_{\mathbf{c}}$ of some $x \in y$ (again, $I(y)$ is uniquely determined up to inner automorphism). We furthermore define

$$
M(Z)=\{(y, \sigma) \mid y \in \mathcal{O}(Z) \text { and } \sigma \in \operatorname{Irr}(I(y))\} .
$$

Regarding $Z$ as a discrete topological space, we may think of $M(Z)$ as the set of isomorphism classes of irreducible $\mathcal{G}_{\mathbf{c}}$-equivariant coherent sheaves on $Z$.

If $Z=\mathcal{G}_{\mathbf{c}}$, then the set $M\left(\mathcal{G}_{\mathbf{c}}\right)$ that we have described coincides with the set $\mathcal{M}\left(\mathcal{G}_{\mathbf{c}}\right)$ introduced by Lusztig in [6]. In loc. cit., he also constructed an explicit embedding of the set of irreducible representations in $\mathbf{c}$ into $\mathcal{M}\left(\mathcal{G}_{\mathbf{c}}\right)$. We denote this map by

$$
\kappa: \operatorname{Irr}(W)_{\mathbf{c}} \hookrightarrow M\left(\mathcal{G}_{\mathbf{c}}\right) .
$$

2.4. A topology on $\bar{A}(C)$ and $\mathcal{G}_{\mathbf{c}}$. Now, fix a unipotent class $C$. In [1], it was shown that the set of conjugacy classes of $\bar{A}(C)$ admits a natural partial order (in which the conjugacy class of the identity is the minimal element), and that $\bar{A}(C)$ has a canonical structure as a Coxeter group up to conjugacy, and therefore a welldefined class of "parabolic subgroups." (It is well known that the set of simple reflections of any irreducible finite Coxeter group is unique up to conjugacy; the point here is that $\bar{A}(C)$ is not, in general, irreducible, but it nevertheless has a canonical decomposition into irreducible factors.) To any element $x \in \bar{A}(C)$, we associate a subgroup $H_{x}$ as follows:

$$
H_{x}=\text { the smallest parabolic subgroup of } \bar{A}(C) \text { containing } x \text {. }
$$

(This definition makes sense because the set of parabolic subgroups is closed under intersection: $H_{x}$ is the intersection of all parabolic subgroups containing $x$.) Evidently, if $x_{1}$ and $x_{2}$ are conjugate, then so are $H_{x_{1}}$ and $H_{x_{2}}$.

We endow $\bar{A}(C)$ with a topology by declaring a set to be open if it is a union of parabolic subgroups. In this topology, for any two elements $x_{1}, x_{2} \in \bar{A}(C)$, we see that the conjugacy class of $x_{1}$ is contained in the closure of the conjugacy class of $x_{2}$ if and only if $H_{x_{2}}$ is conjugate to a subgroup of $H_{x_{1}}$. In particular, the singleton consisting of the identity element is a dense open set.

Next, given a two-sided cell $\mathbf{c}$, recall from [6] that the finite group $\mathcal{G}_{\mathbf{c}}$ can be identified with $\bar{A}\left(C_{\mathbf{c}}\right)$, where $C_{\mathbf{c}}$ is the unique special class in $P_{\mathbf{c}}$. We endow $\mathcal{G}_{\mathbf{c}}$ with a topology as above, and we say that a $\mathcal{G}_{\mathbf{c}}$-equivariant sheaf $\mathcal{F}$ on $\mathcal{G}_{\mathbf{c}}$ is locally trivial if, for each parabolic subgroup $H \subset \mathcal{G}_{\mathbf{c}}$, the action of $H$ on $\mathcal{F}(H)$ is trivial. The stalk at any point $x$ of a locally trivial $G$-equivariant sheaf then carries an action of $\mathcal{G}_{\mathbf{c}}^{x} /\left(H_{x} \cap \mathcal{G}_{\mathbf{c}}^{x}\right)$.

For any conjugacy class $y \in \mathcal{O}\left(\mathcal{G}_{\mathbf{c}}\right)$, we define

$$
\bar{I}(y)=\mathcal{G}_{\mathbf{c}}^{x} /\left(H_{x} \cap \mathcal{G}_{\mathbf{c}}^{x}\right) \simeq N\left(H_{x}\right) / H_{x} \quad \text { for some } x \in y ;
$$

as with $I(y)$, this group is determined independently of the choice of $x$ up to inner automorphism. Next, given a $\mathcal{G}_{\mathbf{c}}$-stable subset $Z \subset \mathcal{G}_{\mathbf{c}}$, the set of isomorphism 
classes of irreducible locally trivial $G$-equivariant sheaves on $Z$ is parametrized by the set

$$
M^{0}(Z)=\{(y, \sigma) \mid y \in \mathcal{O}(Z) \text { and } \sigma \in \operatorname{Irr}(\bar{I}(y))\} .
$$

2.5. Statement of the main result. We regard the set $\mathcal{O}\left(P_{\mathbf{c}}\right)$ as being partially ordered in the usual way: $C_{1} \leq C_{2}$ if $C_{1} \subset \overline{C_{2}}$. In addition, we also endow $\mathcal{O}\left(\mathcal{G}_{\mathbf{c}}\right)$ with an analogous partial order, using the topology defined above.

Theorem 2.1. Let $\mathbf{c}$ be a two-sided cell in $W$.

(1) There is an order-preserving injective map $t: \mathcal{O}\left(P_{\mathbf{c}}\right) \rightarrow \mathcal{O}\left(\mathcal{G}_{\mathbf{c}}\right)$, characterized by the property that $(t(C), 1)=\kappa\left(\chi_{C, 1}\right)$. Moreover, the image of $t$ consists of the set of conjugacy classes of a subgroup $\mathcal{G}_{\mathbf{c}}^{\prime} \subset \mathcal{G}_{\mathbf{c}}$.

(2) For each $C \in \mathcal{O}\left(P_{\mathbf{c}}\right)$, there is a canonical isomorphism $\bar{A}(C) \simeq \bar{I}(t(C))$, and hence a canonical bijection $T: X^{0}\left(P_{\mathbf{c}}\right) \rightarrow M^{0}\left(\mathcal{G}_{\mathbf{c}}^{\prime}\right)$.

(3) The following diagram commutes:

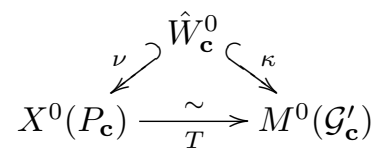

Proof for the exceptional types. Parts (1) and (2) of this theorem were already proved in Theorem 0.4 and Proposition 0.7 of [8]. (Proposition 0.7(b) of loc. cit. asserts that the group we have called $\bar{I}\left(t(C)\right.$ ) (there, $N\left(H_{C}\right) / H_{C}$ ) is isomorphic to $A(C)$, not $\bar{A}(C)$. However, that statement is only meant to apply to nonspecial classes in the exceptional groups. By comparing the tables in [9] with those in [3], one verifies that for all such classes, it is in fact the case that $A(C)=\bar{A}(C)$.)

Only the commutativity of the diagram in part (3) remains. For this, one undertakes the laborious task of comparing tables for the Springer correspondence, in, say, [3], with Lusztig's enumeration of values of $\kappa$ in [6].

\section{The Classical types: Outline}

The proof of the main theorem for the classical types comes down to calculations with various combinatorial objects that parametrize $\operatorname{Irr}(W), X(\mathcal{U})$, and $X\left(\mathcal{G}_{\mathbf{c}}\right)$. The details differ in the three types, but they can all be placed in a common framework, which we will now develop.

3.1. $\bar{A}(C)$ for classical groups. Recall that in the classical groups, the groups $\mathcal{G}_{\mathbf{c}}$ are always products of copies of $\mathbb{Z} / 2 \mathbb{Z}$. In particular, they are always abelian, so the set $\operatorname{Irr}\left(\mathcal{G}_{\mathbf{c}}\right)$ can be identified with the group $\hat{\mathcal{G}}_{\mathbf{c}}=\operatorname{Hom}\left(\mathcal{G}_{\mathbf{c}}, \mathbb{C}^{\times}\right)$, and $M\left(\mathcal{G}_{\mathbf{c}}\right)$ can, in turn be identified with $\mathcal{G}_{\mathbf{c}} \times \hat{\mathcal{G}}_{\mathbf{c}}$. In this section, we will give a particular realization of these spaces that is well-suited to the combinatorics that will follow, and, furthermore, we describe the sets $\operatorname{Irr}\left(\mathcal{G}_{\mathbf{c}} / H_{x}\right)$ with respect to this realization.

Suppose $\mathcal{G}_{\mathbf{c}} \simeq(\mathbb{Z} / 2 \mathbb{Z})^{f}$. Let $V_{\mathbf{c}}$ be the quotient of the $(2 f+1)$-dimensional $\mathbb{F}_{2}$-vector space with basis

$$
e_{0}, e_{1}, \ldots, e_{2 f}
$$

by the relation

$$
e_{0}+e_{2}+e_{4}+\cdots+e_{2 f}=0
$$


(so $\operatorname{dim} V_{\mathbf{c}}=2 f$ ). We endow $V$ with a nondegenerate bilinear form given by

$$
\left\langle e_{i}, e_{j}\right\rangle= \begin{cases}1 & \text { if }|i-j|=1 \\ 0 & \text { otherwise }\end{cases}
$$

(Note that this is consistent with the relation (1).) We make the identification

$$
\mathcal{G}_{\mathbf{c}}=\operatorname{span}\left\{e_{1}, e_{3}, \ldots, e_{2 f-1}\right\},
$$

and we remark, in particular, that the canonical Coxeter generators referred to in Section 2.4 are precisely the elements $e_{1}, e_{3}, \ldots, e_{2 f-1}$. Then, via the bilinear form $\langle$,$\rangle , we can also identify$

$$
\hat{\mathcal{G}}_{\mathbf{c}}=\operatorname{Hom}\left(\mathcal{G}_{\mathbf{c}}, \mathbb{C}^{\times}\right)=\operatorname{span}\left\{e_{0}, e_{2}, \ldots, e_{2 f}\right\} .
$$

Now, let $x \in \mathcal{G}_{\mathbf{c}}$. There is a set of integers $n(x) \subset\{1,3 \ldots, 2 f-1\}$ such that

$$
x=\sum_{j \in n(x)} e_{j} .
$$

Evidently, the subgroup $H_{x}$ is simply the span of $\left\{e_{i} \mid i \in n(x)\right\}$. To describe $\operatorname{Irr}\left(\mathcal{G}_{\mathbf{c}} / H_{x}\right)$ as a subset of $\hat{\mathcal{G}}_{\mathbf{c}}$, we need a slightly refined description of $n(x)$. An element $m \in \mathcal{G}_{\mathbf{c}}$ is called a component of $x$ if it is of the form

$$
m=e_{i}+e_{i+2}+\cdots+e_{i+2 k}
$$

where

$$
\{i, i+2, \ldots, i+2 k\} \subset n(x), \quad i-2 \notin n(x), \quad i+2 k+2 \notin n(x) .
$$

Clearly, $x$ is the sum of its components; each $e_{j}$ appears in at most one component; and the inner product of distinct components is 0 . Next, an element $\lambda \in \hat{\mathcal{G}}_{\mathbf{c}}$ is called a block of $x$ if it is of the form

$$
\lambda=e_{i}+e_{i+2}+\cdots+e_{i+2 k},
$$

and, in addition, the element $m=e_{i+1}+e_{i+3}+\cdots+e_{i+2 k-1} \in \mathcal{G}_{\mathbf{c}}$ is either zero or a component of $x$. (It is permitted that $k=0$, so a single term $e_{i}$ is a block if $i$ is even and $i-1, i+1 \notin n(x)$.) In this case, we say that $m$ is the component associated to $\lambda$.

Note that every $e_{i}$ with $i$ even appears in exactly one block of $x$. Thus the sum of blocks is 0 (by (1)), and any proper subset of the blocks is linearly independent.

Lemma 3.1. $\operatorname{Irr}\left(\mathcal{G}_{\mathbf{c}} / H_{x}\right)$ is the subspace of $\hat{\mathcal{G}}_{\mathbf{c}}$ spanned by the blocks of $x$.

Proof. Let $\lambda$ be a block of $x$, and let $m$ be its associated component. It is clear that $\left\langle\lambda, e_{j}\right\rangle=0$ for each $e_{j}$ appearing in $m$. Moreover, neither $i-1$ nor $i+2 k+1$ belongs to $n(x)$, so in fact, $\left\langle\lambda, e_{j}\right\rangle=0$ for all $j \in n(x)$. Thus $\left.\lambda\right|_{H_{x}}$ is indeed trivial.

Conversely, let $\mu \in \hat{\mathcal{G}}_{\mathbf{c}}$ be such that some, but not all, of the $e_{j}$ appearing in $\lambda$ appear in $\mu$. In other words, $\mu$ is not in the span of the blocks of $x$. Find a pair of consecutive terms in $\lambda$, say $e_{2 p}$ and $e_{2 p+2}$, such that exactly one of them appears in $\mu$. Then $2 p+1 \in n(x)$, yet it is clear that $\left\langle\mu, e_{2 p+1}\right\rangle=1$. Thus $\left.\mu\right|_{H_{x}} \neq 0$. 
3.2. Symbols and $u$-symbols. The combinatorial objects with which all our calculations in the classical types will be carried out are called symbols and u-symbols. Both of these are certain finite sequences $\left(a_{0}, a_{1}, \ldots, a_{r}\right)$ of nonnegative integers, satisfying various additional conditions (that are different for symbols and for $u$ symbols). The value of $r$ (i.e., the length of the sequences), as well as the precise form of the additional conditions, depend on the type. In each type, there is a bijection

$$
i:\{u \text {-symbols }\} \rightarrow\{\text { symbols }\},
$$

and both sets parametrize $\operatorname{Irr}(W)$.

Remark 3.2. Our usage of the terms "symbol" and " $u$-symbol" is close to that of [8], but narrower than the usage elsewhere in the literature. The definitions that we will give correspond to what other authors refer to as symbols or $u$-symbols "of defect 1" (for types $B$ and $C$ ) or "of defect 0 " (for type $D$ ).

Also, symbols and $u$-symbols are both usually written as arrays consisting of two rows of numbers. Here, following [8], we will write them simply as finite sequences, in which the even-numbered entries $a_{0}, a_{2}, \ldots$ may be thought of as the "upper row", and the odd-numbered entries as the "lower row."

Many of the combinatorial results we require will be easier to state and prove if we work with the indices of entries in a symbol or $u$-symbol rather than the entries themselves: that is, we make statements in terms of subsets of $\{0, \ldots, r\}$ rather than subsequences of $\left(a_{0}, \ldots, a_{r}\right)$. The following notation will be particularly useful:

$$
[k, l]=\{k, k+1, \ldots, l\} .
$$

Definition 3.3. Let $\mathbf{a}=\left(a_{0}, \ldots, a_{r}\right)$ be a $u$-symbol, and let $\mathbf{a}^{\prime}=i(\mathbf{a})=\left(a_{0}^{\prime}, \ldots, a_{r}^{\prime}\right)$ be the corresponding symbol.

A number $k \in[0, r]$ is called an isolated point (of $\mathbf{a}$ or $\mathbf{a}^{\prime}$ ) if $a_{k}^{\prime} \neq a_{l}^{\prime}$ for all $l \neq k$.

A subset $[k, l] \subset[0, r]$ is called a ladder of $\mathbf{a}$ if, for some $a$, we have

$$
\begin{gathered}
\left(a_{k}, a_{k+1}, \ldots, a_{l}\right)=(a, a+1, \ldots, a+l-k), \\
a_{k-1}<a-1 \text { if } k>0, \quad \text { and } \quad a_{l+1}>a+l-k+1 \text { if } l<r .
\end{gathered}
$$

A subset $[k, l] \subset[0, r]$ is called a staircase of a if, for some $a$, we have

$$
\begin{gathered}
\left(a_{k}, a_{k+1}, \ldots, a_{l}\right)=(a, a, a+2, a+2, \ldots, a+l-k-1, a+l-k-1), \\
a_{k-1}<a-1 \text { if } k>0, \quad \text { and } \quad a_{l+1}>a+l-k+1 \text { if } l<r .
\end{gathered}
$$

(Note that $l-k+1$ is necessarily even if $[k, l]$ is a staircase.)

A set $[k, l]$ that is either a ladder or a staircase is called a part. The number $k$ is called the bottom of the part; $l$ is called the top; and the length of the part is defined to be $l-k+1$.

It is clear that any two parts of a $u$-symbol must be disjoint. It will follow from the specific definitions of $u$-symbols in each type that in fact the full set of indices $[0, r]$ is always the disjoint union of all the parts.

Symbols and $u$-symbols both fall into families determined by their entries.

Definition 3.4. Two symbols $\mathbf{a}$ and $\mathbf{b}$ are said to be congruent if they contain the same entries (with the same multiplicities), possibly in different orders. A symbol $\left(a_{0}, \ldots, a_{r}\right)$ is special if $a_{0} \leq \cdots \leq a_{r}$. 
Two $u$-symbols $\mathbf{a}$ and $\mathbf{b}$ are said to be similar if they contain the same entries (with the same multiplicities), possibly in different orders. A $u$-symbol $\left(a_{0}, \ldots, a_{r}\right)$ is distinguished if $a_{0} \leq \cdots \leq a_{r}$.

In particular, each congruence class of symbols (resp. similarity class of $u$ symbols) contains a unique special symbol (resp. distinguished $u$-symbol). We now describe a construction that will have multiple uses in the sequel.

Definition 3.5. Let $\mathbf{a}=\left(a_{0}, \ldots, a_{r}\right)$ be a symbol (resp. $u$-symbol), and let $\mu$ be a subset of $\{0, \ldots, r\}$, such that for each $i \in \mu$, the entry $a_{i}$ is distinct from all other entries of $\mathbf{a}$. Suppose there exists a symbol (resp. $u$-symbol) b which contains the same entries as $\mathbf{a}$, but in a different order: namely, we require the positions of a given element in $\mathbf{a}$ and in $\mathbf{b}$ have opposite parity if its position in $\mathbf{a}$ belongs to $\mu$, and the same parity otherwise. (For instance, if $i \in \mu$ is odd, then the number $a_{i}$ should occupy an even-numbered position in b.) When such a symbol (resp. $u$ symbol) exists, it turns out that it is unique. We say that $\mathbf{b}$ is obtained by twisting $\mathbf{a}$ by $\mu$, and we denote it by $\mathbf{b}=\mathbf{a}^{\mu}$.

Thus, the twisting operation preserves congruence classes of symbols and similarity classes of $u$-symbols.

3.3. Symbols, cells, and $\kappa$. Following [5], we know that two symbols are similar if and only if they correspond to representations of $W$ lying in the same two-sided cell. Moreover, the symbols we have called "special" are precisely those corresponding to special representations of $W$.

Now, note that any two congruent symbols have the same set of entries at isolated points, so there is a natural bijection between their respective sets of isolated points.

Definition 3.6. Let $\mathbf{a}_{0}$ is a special symbol, and let $\mathbf{a}$ be a symbol in its congruence class. An isolated point of $\mathbf{a}$ is said to be displaced if it has parity opposite to that of the corresponding isolated point of $\mathbf{a}_{0}$. Every symbol has an even number of displaced isolated points.

Later, in each classical type, we will obtain a more direct characterization of displaced isolated points.

Let $\tilde{V}\left(\mathbf{a}_{0}\right)$ be the power set of the set of isolated points, regarded as an $\mathbb{F}_{2^{-}}$ vector space with addition given by symmetric difference of sets. Let $V\left(\mathbf{a}_{0}\right)$ be the subspace of $\tilde{V}\left(\mathbf{a}_{0}\right)$ consisting of sets with even cardinality. We endow $V\left(\mathbf{a}_{0}\right)$ with a nondegenerate symmetric bilinear form by putting $\langle v, w\rangle=|v \cap w| \bmod 2$. (Here $|v \cap w|$ is the cardinality of the set $v \cap w$.)

Since any symbol has an even number of displaced isolated points, there is a natural map

$$
\tilde{\kappa}:\left\{\begin{array}{c}
\text { symbols } \\
\text { congruent to } \mathbf{a}_{0}
\end{array}\right\} \rightarrow V\left(\mathbf{a}_{0}\right) \quad \text { by } \quad \mathbf{a} \mapsto\left\{\begin{array}{c}
\text { displaced } \\
\text { isolated points of } \mathbf{a}
\end{array}\right\}
$$

Now, following [6], if $\mathbf{c}$ is the two-sided cell corresponding to the special symbol $\mathbf{a}_{0}$, then there is a natural surjective map $\pi$ from $V\left(\mathbf{a}_{0}\right)$ to the space $V_{\mathbf{c}}$ of Section 3.1; in fact, it is an isomorphism except in type $D$. We will describe this map explicitly in each type by labelling certain elements of $V\left(\mathbf{a}_{0}\right)$ with the names $e_{0}, e_{1}, \ldots, e_{2 f}$. With this in mind, the map $\tilde{\kappa}$ defined above can be regarded as a combinatorial version of Lusztig's map $\kappa: \operatorname{Irr}(W)_{\mathbf{c}} \rightarrow V_{\mathbf{c}}=M\left(\mathcal{G}_{\mathbf{c}}\right)$, in that the 
following diagram commutes:

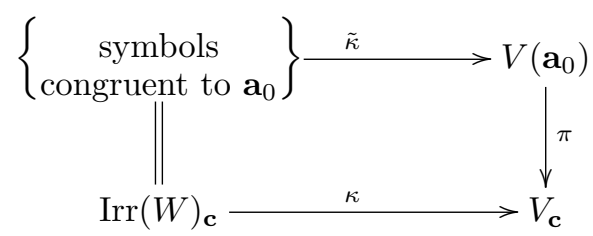

It is not difficult to see that if $\mathbf{a}$ is a symbol and $\mu$ is a set consisting of an even number of isolated points, then $\tilde{\kappa}\left(\mathbf{a}^{\mu}\right)=\tilde{\kappa}(\mathbf{a})+\mu$ (assuming $\mathbf{a}^{\mu}$ is defined). A more significant observation is that, by commutativity of the above diagram, we have

$$
\kappa\left(\mathbf{a}^{\mu}\right)=\kappa(\mathbf{a})+\pi(\mu) .
$$

3.4. $u$-Symbols and the Springer correspondence. Following [7], we know that two $u$-symbols are similar if and only if the Springer correspondence maps the corresponding representations to local systems on the same unipotent class. Moreover, the distinguished $u$-symbols are those corresponding to Springer representations (that is, representations mapped to the trivial local system on some unipotent class). There is thus a bijection between distinguished $u$-symbols and unipotent classes.

Given a distinguished $u$-symbol $\mathbf{a}_{0}=\left(a_{0}, \ldots, a_{r}\right)$, let $H(\mathbf{a})$ be the power set of the set of ladders of $\mathbf{a}$, regarded as an $\mathbb{F}_{2}$-vector space with addition given by symmetric difference of sets.

Let $C$ be the unipotent class corresponding to a. In each classical type, there is a surjective map $p: H(\mathbf{a}) \rightarrow \operatorname{Irr}(A(C))$, such that the set of all ladders lies in its kernel. (To be more precise, $\operatorname{Irr}(A(C))$ can be canonically identified with a quotient of some subspace of $H(\mathbf{a})$, as described in [3]. The map $p$ may not be canonically determined, but we may choose it so that its kernel contains the set of all ladders.)

By abuse of language, we will often regard an element $\mu \in H(\mathbf{a})$ as a subset of $[0, r]$, rather than as a set of ladders, by replacing $\mu$ by the union of its members. This abuse allows us to speak of twisting a $u$-symbol by an element of $H(\mathbf{a})$.

It turns out that all $u$-symbols in the similarity class of a arise by twisting a by a suitable set of ladders. Moreover, this set of ladders is uniquely determined. Given a $u$-symbol $\mathbf{a}^{\prime}$ similar to $\mathbf{a}$, we denote by $\tilde{\nu}\left(\mathbf{a}^{\prime}\right) \in H(\mathbf{a})$ the unique element such that $\mathbf{a}^{\prime}=\mathbf{a}^{\tilde{\nu}\left(\mathbf{a}^{\prime}\right)}$. The following analogue of (2) commutes, so $\tilde{\nu}$ can be thought of as a combinatorial version of the Springer correspondence:

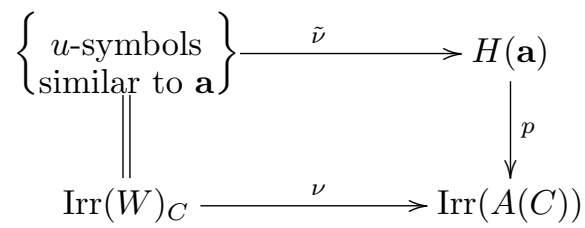

(Here $\operatorname{Irr}(W)_{C} \subset \operatorname{Irr}(W)$ is just the set of representations to which $\nu$ assigns local systems on $C$.) In addition, we also have an analogue of (3):

$$
\nu\left(\mathbf{a}^{\mu}\right)=(C, p(\mu))
$$

whenever $\mathbf{a}^{\mu}$ is defined.

We will also introduce subsets of $[0, r]$ called blocks, which are certain unions of ladders and staircases. Let $\bar{H}(\mathbf{a})$ be the power set of the set of blocks. By assigning 
to a block the set of ladders contained within it, we obtain an injective map from $\bar{H}(\mathbf{a})$ to $H(\mathbf{a})$. In fact, we will regard $\bar{H}(\mathbf{a})$ as a subset of $H(\mathbf{a})$ via this map.

3.5. Isolated points and blocks. In this section, we give an outline of the proof of Theorem 2.1 in the classical types. Specifically, we state without proof a number of intermediate lemmas and propositions, and indicate how these statements together imply the theorem. The proofs of the intermediate results are slightly different in each type, and depend on the details of the combinatorial definitions of symbols and $u$-symbols.

We fix the following notations: let $\mathbf{a}$ be a distinguished $u$-symbol, and let $\mathbf{a}_{0}$ be the unique $u$-symbol such that $i\left(\mathbf{a}_{0}\right)$ is special and congruent to $i(\mathbf{a})$. (Note that $\mathbf{a}_{0}$ is necessarily distinguished, since all special representations of $W$ are Springer representations.) Let $\mathbf{c}$ be the two-sided cell corresponding to $i\left(\mathbf{a}_{0}\right)$, and let $C$ be the unipotent class corresponding to $\mathbf{a}$.

The first result tells us where isolated points occur in a $u$-symbol:

Proposition 3.7. Let a be a distinguished u-symbol. The top and bottom of each block of a are nondisplaced isolated points. In addition to these, each block contains an even number (possibly zero) of additional isolated points, all of which are displaced.

We remark that the terms "top" and "bottom" as applied to blocks are not quite the same as for ladders and staircases - the details vary by type and will be given in Section 4. In particular, not every block has both a top and a bottom, so this proposition does not imply that there are in all an even number of isolated points.

Given a block $b$ of a distinguished $u$-symbol $\mathbf{a}$, let $\lambda_{b} \in V\left(\mathbf{a}_{0}\right)$ be the set of all isolated points in $b$, and let $m_{b} \in V_{\mathbf{c}}$ be the set of displaced isolated points of $b$ (here $\mathbf{c}$ is the two-sided cell corresponding to the congruence class of $i(\mathbf{a})$ ). In other words, $m_{b}$ is obtained from $\lambda_{b}$ by omitting the top and bottom of $b$.

Note that according to the preceding lemma, $\tilde{\kappa}(\mathbf{a})=\sum m_{b}$, where $\mathbf{a}$ is a distinguished $u$-symbol, and $b$ runs over the blocks of $\mathbf{a}$.

Proposition 3.8. For any block $b$ of $\mathbf{a}$, the element $\pi\left(m_{b}\right) \in \mathcal{G}_{\mathbf{c}} \times \hat{\mathcal{G}}_{\mathbf{c}}$ is of the form $(x, 1), x \in \mathcal{G}_{\mathbf{c}}$.

As a consequence of this proposition, we recover Lusztig's result [8] that $\kappa\left(\chi_{C, 1}\right)$ is of the form $(x, 1)$ for some $x \in \mathcal{G}_{\mathbf{c}}$. In particular, we henceforth have the map $t: \mathcal{O}\left(P_{\mathbf{c}}\right) \rightarrow \mathcal{O}\left(\mathcal{G}_{\mathbf{c}}\right)$ available to us.

Proposition 3.9. If $C_{1}$ and $C_{2}$ are two unipotent classes in the same special piece, then $t\left(C_{1}\right) \leq t\left(C_{2}\right)$ in the partial order on $\mathcal{G}_{\mathbf{c}}$ if and only if $C_{1}$ is in the closure of $\mathrm{C}_{2}$.

Once this assertion is established, we will have completed the proof of part 1 of Theorem 2.1.

Proposition 3.10. The elements $\pi\left(m_{b}\right)$, as b runs over the blocks of $\mathbf{a}$, are the components of $t(C)$, and the elements $\pi\left(\lambda_{b}\right)$ are the blocks of $t(C)$.

Since the blocks of a are a basis for $\bar{H}(\mathbf{a})$, and $\operatorname{Irr}\left(\mathcal{G}_{\mathbf{c}} / H_{x}\right)$ is generated by the blocks of $x$, we see that the map $b \mapsto \lambda_{b}$ induces a surjective map $\bar{H}(\mathbf{a}) \rightarrow$ $\operatorname{Irr}\left(\mathcal{G}_{\mathbf{c}} / H_{t(C)}\right)=\operatorname{Irr}(\bar{I}(t(C)))$. The next statement lets us study this surjection more carefully. 
Proposition 3.11. The kernel of the restriction $\left.p\right|_{\bar{H}(\mathbf{a})}: \bar{H}(\mathbf{a}) \rightarrow \operatorname{Irr}(A(C))$ is generated by the sum of all the blocks of $\mathbf{a}$.

(Since $\bar{A}(C)$ is a quotient of $A(C), \operatorname{Irr}(\bar{A}(C))$ is naturally a subset of $\operatorname{Irr}(A(C))$.) Recall, from the remark immediately preceding Lemma 3.1, that the sum of all blocks of $t(C)$ is 0 in $V_{\mathbf{c}}$. Therefore, the map $\left.p\right|_{\bar{H}(\mathbf{a})}: \bar{H}(\mathbf{a}) \rightarrow \operatorname{Irr}(A(C))$ factors through the natural surjection $\bar{H}(\mathbf{a}) \rightarrow \bar{I}(t(C))$, and induces an injective map $\operatorname{Irr}(\bar{I}(t(C))) \hookrightarrow \operatorname{Irr}(A(C))$.

However, to establish the commutativity of the diagram in part 3, we need a slightly stronger statement.

Proposition 3.12. If $\mathbf{a}$ is a distinguished $u$-symbol and $b$ is a block, then $i\left(\mathbf{a}^{b}\right)=$ $(i(\mathbf{a}))^{\lambda_{b}}$.

Proposition 3.13. The image of $\left.p\right|_{\bar{H}(\mathbf{a})}: \bar{H}(\mathbf{a}) \rightarrow \operatorname{Irr}(A(C))$ is precisely $\operatorname{Irr}(\bar{A}(C))$.

An immediate consequence of this last proposition is that the image of the aforementioned injective map $\operatorname{Irr}(\bar{I}(t(C))) \hookrightarrow \operatorname{Irr}(A(C))$ is $\operatorname{Irr}(\bar{A}(C))$. Part 2 of Theorem 2.1 is thus established; the map $T: X^{0}\left(P_{\mathbf{c}}\right) \rightarrow M^{0}\left(\mathcal{G}_{\mathbf{c}}^{\prime}\right)$ is described by $T(C, p(b))=\left(t(C), \pi\left(\lambda_{b}\right)\right)$.

Finally, let us translate Proposition 3.12 into representation-theoretic terms. From the equality (5), we know that $\mathbf{a}^{b}$ is the $u$-symbol for $\chi_{C, p(b)}$. On the other hand, we know that $\kappa(i(\mathbf{a}))=(t(C), 1)$, so from (3), we have $\kappa\left(i(\mathbf{a})^{\lambda_{b}}\right)=$ $\left(t(C), \pi\left(\lambda_{b}\right)\right)$. In other words, we have $\kappa\left(\chi_{C, p(b)}\right)=\left(t(C), \pi\left(\lambda_{b}\right)\right)=T(C, p(b))$. Therefore,

$$
\kappa(\chi)=T(\nu(\chi)) \quad \text { for all } \chi \in \operatorname{Irr}(W)_{\mathbf{c}}^{0} .
$$

This establishes the commutativity of the diagram in part 3 of Theorem 2.1. The proof of the theorem is thus completed.

\section{The Classical types: Combinatorics}

It remains, of course, to fill in a number of details separately in each of the classical types. The missing ingredients are as follows:

(1) Definitions of "symbol," " $u$-symbol," and "block."

(2) A careful study of where isolated points may occur.

(3) Proofs of Propositions 3.7-3.13.

Below, we give full details for each of these steps in type $C$. In particular, item 2 is treated in Lemmas 4.2 and 4.3. However, types $B$ and $D$ receive a much more cursory treatment: we provide the requisite definitions, and we state without proof the necessary results on isolated points (see Lemmas 4.12 and 4.13 for type $B$, and Lemmas 4.15 and 4.16 for type $D$ ), but we make no comment at all on Propositions 3.7-3.13. All the missing proofs in types $B$ and $D$ are, of course, quite similar to the corresponding proofs in type $C$.

4.1. Type $C$. Let $\Psi_{2 n, m}^{\prime}$ (resp. $\left.\tilde{\Phi}_{2 n, m}^{\prime}\right)$ be the set of sequences $\mathbf{a}=\left(a_{0}, a_{1}, \ldots, a_{2 m}\right)$ satisfying the following conditions:

$$
\begin{aligned}
& 0 \leq a_{0}, \quad 1 \leq a_{1}, \quad a_{i} \leq a_{i+2}-2, \quad \sum a_{i}=n+2 m^{2}+m \quad \text { for } \mathbf{a} \in \Psi_{2 n, m}^{\prime}, \\
& 0 \leq a_{0}, \quad 0 \leq a_{1}, \quad a_{i} \leq a_{i+2}-1, \quad \sum a_{i}=n+m^{2} \quad \text { for } \mathbf{a} \in \Phi_{2 n, m}^{\prime}
\end{aligned}
$$


There are embeddings $S: \Psi_{2 n, m}^{\prime} \rightarrow \Psi_{2 n, m+1}^{\prime}, S: \Phi_{2 n, m}^{\prime} \rightarrow \Phi_{2 n, m+1}^{\prime}$ given by

$$
\begin{array}{ll}
S(\mathbf{a})=\left(0,1, a_{0}+2, \ldots, a_{2 m}+2\right) & \text { for } \mathbf{a} \in \Psi_{2 n, m}^{\prime}, \\
S(\mathbf{a})=\left(0,0, a_{0}+1, \ldots, a_{2 m}+1\right) & \text { for } \mathbf{a} \in \Phi_{2 n, m}^{\prime} .
\end{array}
$$

These maps are called shift operations. Put $\Psi_{2 n}^{\prime}=\lim _{m \rightarrow \infty} \Psi_{2 n, m}^{\prime}$ and $\Phi_{2 n}^{\prime}=$ $\lim _{m \rightarrow \infty} \Phi_{2 n, m}^{\prime}$. These sets are finite; We fix an $m$ large enough that $\Psi_{2 n}^{\prime} \simeq \Psi_{2 n, m}^{\prime}$ and $\Phi_{2 n}^{\prime} \simeq \Phi_{2 n, m}^{\prime}$. Elements of $\Phi_{2 n}^{\prime}$ (resp. $\Psi_{2 n}^{\prime}$ ) are called symbols (resp. u-symbols).

The bijection $i: \Psi_{2 n}^{\prime} \rightarrow \Phi_{2 n}^{\prime}$ is given by

$$
i\left(a_{0}, \ldots, a_{2 m}\right)=\left(a_{0}, a_{1}-1, a_{2}-1, \ldots, a_{2 m}-m\right) .
$$

Definition 4.1. A block is a subset $[k, l]$ of $[0,2 m]$ that meets one of the following conditions:

- $[k, l]$ is a union of consecutive parts $P_{1}, \ldots, P_{r}$, where $P_{1}$ and $P_{r}$ are ladders, $P_{r}$ is the unique part with even top, and $P_{1}$ is the unique part with odd bottom.

- $k=0$, and $[0, l]$ is the union of consecutive parts $P_{2}, \ldots, P_{r}$, where $P_{r}$ is a ladder and the only part with even top, and where all parts have even bottom.

In either case, the top of the block is the top of $P_{r}$, and its bottom is the bottom of $P_{1}$, provided the latter is defined. That is, blocks meeting the second condition above are said to have no bottom.

We remark that it is permitted that $l=1$, in which case the block consists of a single ladder. A part belongs to some block if and only if it is not a staircase with odd bottom (and hence even top).

Lemma 4.2. For any $\mathbf{a} \in \Psi_{2 n}^{\prime}$, there are an odd number of isolated points. Suppose $i(\mathbf{a})=\left(a_{0}^{\prime}, \ldots, a_{2 m}^{\prime}\right)$, and let $k_{0}, \ldots, k_{2 f}$ be the isolated points, numbered such that

$$
a_{k_{0}}^{\prime}<a_{k_{1}}^{\prime}<\cdots<a_{k_{2 f}}^{\prime} .
$$

If $\mathbf{a}$ is special, then $k_{t} \equiv t(\bmod 2)$.

We will adhere to the convention introduced in this lemma for naming the isolated points. In particular, this means that if $\mathbf{a}$ is not special, it is not necessarily the case that $k_{t}<k_{t+1}$.

If $\mathbf{a}_{0}$ is a special symbol or $u$-symbol, we identify $V\left(\mathbf{a}_{0}\right)$ with the space $V_{\mathbf{c}}$ of Section 3.1 by

$$
e_{i}=\left\{k_{i-1}, k_{i}\right\}, \quad i=1, \ldots, 2 f ; \quad e_{0}=\left\{k_{1}, k_{2}, \ldots, k_{2 f}\right\} .
$$

Lemma 4.3. Let $\mathbf{a}=\left(a_{0}, \ldots, a_{2 m}\right)$ be a distinguished $u$-symbol, and let $\mathbf{a}^{\prime}=i(\mathbf{a})=$ $\left(a_{0}^{\prime}, \ldots, a_{2 m}\right)$ be the corresponding symbol. Let $k$ and $l$ be two integers such that $0 \leq k<l \leq 2 m$.

(1) If $k$ and $l$ belong to distinct parts, then $a_{k}^{\prime} \neq a_{l}^{\prime}$.

(2) If $[k, l]$ is a staircase with even bottom, then $k+1$ and $l-1$ are both isolated points. A staircase with odd bottom contains no isolated points.

(3) If $[k, l]$ is a ladder, then $k$ is an isolated point if it is odd, and $l$ is an isolated point if it is even. There are no other isolated points in $[k, l]$.

(4) The number of isolated points in any part is congruent to the length of the part modulo 2. 
(5) For each $t$, either $k_{t}<k_{t+1}$, or $k_{t}=k_{t+1}+1$ and $\left[k_{t+1}, k_{t}\right]$ is a staircase with even bottom. In this case, $k_{t}$ and $k_{t+1}$ are both displaced.

(6) Displaced isolated points occur in pairs $\left\{k_{t}, k_{t+1}\right\}=e_{t+1}$ with $t$ even, with one such pair for each staircase with even bottom.

Proof. (1) If $k \equiv l(\bmod 2)$ but $k \neq l$, it follows from the definition of $\Phi_{2 n}^{\prime}$ that $a_{k}^{\prime} \neq a_{l}^{\prime}$. Indeed, the fact that $a_{i}^{\prime} \leq a_{i+2}^{\prime}-1$ implies that $a_{i} \leq a_{i+2 j}-j$. We need only treat the case where $k \neq \equiv l(\bmod 2)$.

Assume now that $k<l, k$ is even, and $l$ is odd. Since $a_{l-1} \leq a_{l}, a_{l-1}^{\prime}=$ $a_{l-1}-(l-1) / 2$, and $a_{l}^{\prime}=a_{l}-(l+1) / 2$, it follows that $a_{l}^{\prime} \geq a_{l-1}^{\prime}-1$. Therefore, assuming $k<l-1$, we have

$$
a_{k}^{\prime} \leq a_{l-1}^{\prime}-(l-1-k)<a_{l-1}^{\prime}-1 \leq a_{l}^{\prime} .
$$

If $k=l-1$, however, the fact that $k$ and $l$ are in different parts implies that $a_{l-1} \leq a_{l}-2$, whence $a_{l}^{\prime} \geq a_{l-1}^{\prime}+1=a_{l}^{\prime}+2$. Thus, $a_{k}^{\prime} \neq a_{l}^{\prime}$. The reasoning is similar if $k$ is odd and $l$ is even.

(2) Suppose $\left(a_{k}, a_{k+1}, \ldots, a_{l}\right)=\left(a, a, a+2, a+2, \ldots, a+\frac{l-k+1}{2}\right)$. For $i \in[k, l]$, it is easy to verify that:

$$
a_{i}^{\prime}= \begin{cases}a-k+i / 2 & \text { if } k \text { is even and } i \text { is even, } \\ a-k+(i-3) / 2 & \text { if } k \text { is even and } i \text { is odd, } \\ a-k+(i-2) / 2 & \text { if } k \text { is odd and } i \text { is even, } \\ a-k+(i-1) / 2 & \text { if } k \text { is odd and } i \text { is odd. }\end{cases}
$$

If $k$ is odd, then $a_{i}^{\prime}=a_{i+1}^{\prime}$ for $i=k, k+2, \ldots, l-1$, so there are no isolated points. On the other hand, if $k$ is even, then $a_{i}^{\prime}=a_{i+3}^{\prime}$ for $i=k, k+2, \ldots, l-3$, so none of the integers

$$
k, k+2, \ldots, l-3 ; \quad k+3, k+5, \ldots, l
$$

are isolated. However, $a_{k+1}^{\prime}$ and $a_{l-1}^{\prime}$ are not duplicated. In view of previous statement, we see that $k+1$ and $l-1$ are isolated. have

Suppose $\left(a_{k}, a_{k+1}, \ldots, a_{l}\right)=(a, a+1, \ldots, a+l-k)$. For $i \in[k, l]$, we

$$
a_{i}^{\prime}= \begin{cases}a-k+i / 2 & \text { if } i \text { is even, } \\ a-k+(i-1) / 2 & \text { if } i \text { is odd. }\end{cases}
$$

Thus, $a_{i}^{\prime}=a_{i+1}^{\prime}$ if $i \in[k, l]$ is even. It follows that $k$ is isolated if and only if it is odd, that $l$ is isolated if and only if it is even, and that no other points can be isolated.

(4) From the preceding parts of the lemma, we see that each staircase, and each ladder of even cardinality, contains 0 or 2 isolated points, whereas each ladder of odd cardinality contains exactly one isolated point.

(5) From part 1 we see that the inequality $k_{t}<k_{t+1}$ can be violated only if $k_{t}$ and $k_{t+1}$ belong to the same part. Examining the formulas above for isolated points in ladders and staircases yields the result.

(6) From parts 3 and 4, it follows that no isolated points in ladders can be displaced, while parts 2 and 4 together imply that all isolated points in staircases are displaced.

The following proposition is now an immediate consequence of the definition of blocks and parts (2) and (3) of the preceding lemma. 
Proposition 4.4. Let a be a distinguished u-symbol. The top and bottom of each block of a are nondisplaced isolated points. In addition to these, each block contains an even number (possibly zero) of additional isolated points, all of which are displaced. There are no isolated points that do not belong to any block.

Proposition 4.5. For any block $b$ of a distinguished $u$-symbol $\mathbf{a}$, the element $m_{b} \in$ $\mathcal{G}_{\mathbf{c}} \times \hat{\mathcal{G}}_{\mathbf{c}}$ is of the form $(x, 1), x \in \mathcal{G}_{\mathbf{c}}$.

Proof. It follows immediately from Lemma 4.3(6) that $\kappa(\mathbf{a})$ is a sum of $e_{i}$ 's with $i$ odd. Thus, $\kappa(\mathbf{a}) \in \mathcal{G}_{\mathbf{c}} \times\{1\} \subset M\left(\mathcal{G}_{\mathbf{c}}\right)$.

Proposition 4.6. If $C_{1}$ and $C_{2}$ are two unipotent classes in the same special piece, then $t\left(C_{1}\right) \leq t\left(C_{2}\right)$ in the partial order on $\mathcal{G}_{\mathbf{c}}$ if and only if $C_{1}$ is in the closure of $C_{2}$.

Proof. Let a be a distinguished $u$-symbol corresponding to a unipotent class $C$, and suppose that $k_{t-1}$ and $k_{t}$ are consecutive nondisplaced isolated points of a, with $t$ odd. That is, if we write $\kappa(\mathbf{a})=(x, 1)$, then the basis element $e_{t} \in \mathcal{G}_{\mathbf{c}}$ does not occur in $x$. Now, assume that the twisted symbol $i(\mathbf{a})^{e_{t}}$ is defined, and let $\mathbf{a}^{\prime}$ be the $u$-symbol satisfying $i\left(\mathbf{a}^{\prime}\right)=i(\mathbf{a})^{e_{t}}$. Furthermore, let us assume that $\mathbf{a}^{\prime}$ is also distinguished, corresponding to the unipotent class $C^{\prime}$. (In fact, it is a consequence of later results that $\mathbf{a}^{\prime}$ must be distinguished if it exists.) Evidently, we have $\kappa\left(\mathbf{a}^{\prime}\right)=\left(x+e_{t}, 1\right)$. Now, $x+e_{t}<x$. The first step will be to prove that in this context, $C^{\prime}$ lies in the closure of $C$. We introduce the following notation:

$$
\begin{array}{rlrl}
\mathbf{a} & =\left(a_{0}, \ldots, a_{2 m}\right) \\
\mathbf{a}^{\prime} & =\left(a_{0}^{\prime}, \ldots, a_{2 m}\right) & i(\mathbf{a}) & =\mathbf{b}=\left(b_{0}, \ldots, b_{2 m}\right) \\
i\left(\mathbf{a}^{\prime}\right)=\mathbf{b}^{e_{t}} & =\mathbf{b}^{\prime}=\left(b_{0}^{\prime}, \ldots, b_{2 m}^{\prime}\right)
\end{array}
$$

Now, from the description of isolated points in Lemma 4.3 and Proposition 4.4, we know that $k_{t-1}$ must be the top of a block and $k_{t}$ the bottom of the next block, and therefore that $\left[k_{t-1}+1, k_{t}-1\right]$ is a union of staircases with odd bottom. In particular, $a_{i}=a_{i+1}$ for all odd $i \in\left[k_{t-1}+1, k_{t}-1\right]$, and therefore, from the formula for $i$, we have $b_{i}=b_{i+1}$ for all odd $i \in\left[k_{t-1}+1, k_{t}-1\right]$ as well. (On the other hand, we note for later reference that $a_{i} \leq a_{i+1}-2$ for all even $i \in\left[k_{t-1}, k_{t}\right]$.) Using these observations, one can show that the twisted symbol is described by

$$
b_{i}^{\prime}= \begin{cases}b_{i} & \text { if } i \notin\left[k_{t-1}, k_{t}\right], \\ b_{i+1} & \text { if } i \in\left[k_{t-1}, k_{t}\right] \text { and } i \text { is even, } \\ b_{i-1} & \text { if } i \in\left[k_{t-1}+3, k_{t}\right] \text { and } i \text { is odd. }\end{cases}
$$

(It suffices to verify that the sequence $\left(b_{0}, \ldots, b_{2 m}\right)$ is a valid symbol, and that it satisfies the parity conditions in the definition of twisting.)

Using the formula for $i$ again, we find that

$$
a_{i}^{\prime}= \begin{cases}a_{i} & \text { if } i \notin\left[k_{t-1}, k_{t}\right], \\ a_{i+1}-1 & \text { if } i \in\left[k_{t-1}, k_{t}\right] \text { and } i \text { is even, } \\ a_{i-1}+1 & \text { if } i \in\left[k_{t-1}, k_{t}\right] \text { and } i \text { is odd. }\end{cases}
$$

Now, since $a_{i} \leq a_{i+1}-2$ for all even $i \in\left[k_{t-1}, k_{t}\right]$, we observe in particular that if $i \in\left[k_{t-1}, k_{t}\right]$ is even, then

$$
a_{i}^{\prime}>a_{i}, \quad a_{i+1}^{\prime}<a_{i+1}^{\prime}, \quad a_{i}^{\prime}+a_{i+1}^{\prime}=a_{i}+a_{i+1} .
$$


Now, for any $u$-symbol $\mathbf{c}=\left(c_{0}, \ldots, c_{2 m}\right)$, we define the sums

$$
\sigma_{j}(\mathbf{c})=\sum_{i=j}^{2 m} c_{i} .
$$

From the above formulas, it is easy to see that

$$
\begin{array}{ll}
\sigma_{j}\left(\mathbf{a}^{\prime}\right)<\sigma_{j}(\mathbf{a}) & \text { if } j \in\left[k_{t-1}, k_{t}\right] \text { is odd, } \\
\sigma_{j}\left(\mathbf{a}^{\prime}\right)=\sigma_{j}(\mathbf{a}) & \text { for all other } j \in[0,2 m] .
\end{array}
$$

It was shown in [2, Lemme 3.2] that $C^{\prime}$ is contained in the closure of $C$ if and only if $\sigma_{j}\left(\mathbf{a}^{\prime}\right) \leq \sigma_{j}(\mathbf{a})$ for all $j$. So $C^{\prime}$ is indeed contained in the closure of $C$.

More generally, if $C_{1}$ and $C_{2}$ are two unipotent classes in the same special piece with the property that $t\left(C_{1}\right)<t\left(C_{2}\right)$, then $t\left(C_{1}\right)$ contains various terms $e_{i}$ not appearing in $t\left(C_{2}\right)$. Adding these terms to $t\left(C_{2}\right)$ one at a time and iterating the above argument shows that $C_{1}$ is contained in the closure of $C_{2}$.

It remains to prove the opposite implication. We will show that if $t\left(C_{1}\right)$ and $t\left(C_{2}\right)$ are incomparable, then so are $C_{1}$ and $C_{2}$. Let $\mathbf{a}_{1}$ and $\mathbf{a}_{2}$ be the corresponding distinguished $u$-symbols, and let $\mathbf{a}_{0}$ be the $u$-symbol corresponding to the special class in their special piece. Let $k_{0}, \ldots, k_{2 f}$ be the isolated points of $\mathbf{a}_{0}$. Let $\left\{k_{s-1}, k_{s}\right\}$ be a pair of consecutive isolated points, with $s$ odd, such that the corresponding isolated points of $\mathbf{a}_{1}$ are displaced, but those of $\mathbf{a}_{2}$ are not. In other words, the term $e_{s}$ appears in $t\left(C_{1}\right)$, but not in $t\left(C_{2}\right)$. On the other hand, let $\left\{k_{t-1}, k_{t}\right\}$ be another such pair, with $t$ odd, which is displaced in $\mathbf{a}_{2}$ but not in $\mathbf{a}_{1}$. (Such pairs must exist if $t\left(C_{1}\right)$ and $t\left(C_{2}\right)$ are incomparable.) Choose an odd number $j_{1} \in\left[k_{s-1}, k_{s}\right]$ and another odd number $j_{2} \in\left[k_{t-1}, k_{t}\right]$. The above calculations of $\sigma_{j}$ show that:

$$
\begin{aligned}
& \sigma_{j_{1}}\left(\mathbf{a}_{1}\right)<\sigma_{j_{1}}\left(\mathbf{a}_{0}\right)=\sigma_{j_{1}}\left(\mathbf{a}_{2}\right) \\
& \sigma_{j_{2}}\left(\mathbf{a}_{1}\right)=\sigma_{j_{2}}\left(\mathbf{a}_{0}\right)>\sigma_{j_{2}}\left(\mathbf{a}_{2}\right)
\end{aligned}
$$

Again invoking [2, Lemme 3.2], we see that this pair of inequalities implies that neither $C_{1}$ nor $C_{2}$ may be contained in the closure of the other.

Proposition 4.7. Let a be a distinguished u-symbol, corresponding to the unipotent class $C$. The sets $\lambda_{b}$, as $b$ runs over the blocks of $\mathbf{a}$, are precisely the blocks of $t(C)$; moreover, the associated component of $\lambda_{b}$ is $m_{b}$.

Proof. Let $b$ a block of $\mathbf{a}$, and let $k_{t-1}, k_{t}, \ldots, k_{t+2 j}$ be the isolated points of $b$, where $k_{t-1}$ is the bottom of $b$ and $k_{t+2 j}$ is its top. (We assume here that $b$ has a bottom; later, we will indicate the modifications to the proof that are required when $b$ has no bottom.) Note that $t$ is even. Moreover, $k_{t-2}$ cannot be displaced, because it must be the top of the preceding block, while $k_{t+2 j+1}$ is either undefined or the bottom of next block. Since the pairs $\left\{k_{t-2}, k_{t-1}\right\}$ and $\left\{k_{t+2 j}, k_{t+2 j+1}\right\}$ contain no displaced isolated points, the terms $e_{t-1}$ and $e_{t+2 j+1}$ (provided the latter is defined) do not appear in $\kappa(\mathbf{a})$. Since we evidently have

$$
m_{b}=\left\{k_{t}, \ldots, k_{t+2 j-1}\right\}=e_{t+1}+e_{t+3}+\cdots+e_{t+2 j-1},
$$

we see that if $m_{b}$ is nonzero, then it satisfies the definition of a component of $x$. Moreover, the element

$$
\lambda_{b}=\left\{k_{t-1}, \ldots, k_{t+2 j}\right\}=e_{t}+e_{t+2}+\cdots+e_{t+2 j}
$$

is clearly the block whose associated component is $m_{b}$. 
If $b$ has no bottom, then the isolated points of $b$ are of the form $k_{0}, k_{1}, \ldots, k_{2 j}$. The above formula for $m_{b}$ is still valid, with $t=0$, Recall, however, that the definition of $\lambda_{b}$ is different when $b$ contains an odd number of isolated points: if a contains $2 f+1$ isolated points in all, then

$$
\begin{aligned}
\lambda_{b} & =\left\{k_{2 j+1}, k_{2 j+2}, \ldots, k_{2} f\right\}=e_{2 j+2}+e_{2 j+4}+\cdots+e_{2 f} \\
& =e_{0}+e_{2}+\cdots+e_{2 j} .
\end{aligned}
$$

Again, $\lambda_{b}$ is a block of $t(C)$, and $m_{b}$ is its associated component.

Proposition 4.8. The kernel of the restriction $\left.p\right|_{\bar{H}(\mathbf{a})}: \bar{H}(\mathbf{a}) \rightarrow \operatorname{Irr}(A(C))$ is generated by the sum of all the blocks of $\mathbf{a}$.

Proof. Recall that $p: H(\mathbf{a}) \rightarrow \operatorname{Irr}(A(C))$ was defined such that the set of all ladders is in its kernel. Since every ladder is in a block, it is clear that the set of all blocks is in the kernel of $\left.p\right|_{\bar{H}(\mathbf{a})}$. On the other hand, if $\mu \in \bar{H}(\mathbf{a})$ is any set of blocks not containing the unique block of odd length, it is easy to verify that $\mathbf{a}^{\mu}$ is a welldefined $u$-symbol. Moreover, distinct $\mu$ 's clearly result in distinct twisted $u$-symbols. The injectivity of the Springer correspondence and the commutativity of (4) imply that $\left.p\right|_{\bar{H}(\mathbf{a})}$ must be injective when restricted to the hyperplane consisting of sets of blocks not containing the unique block of odd length. Thus, the set of all blocks generates the kernel of $\left.p\right|_{\bar{H}(\mathbf{a})}$.

Proposition 4.9. If $\mathbf{a}$ is a distinguished $u$-symbol and $b$ is a block, then $i\left(\mathbf{a}^{b}\right)=$ $(i(\mathbf{a}))^{\lambda_{b}}$.

Proof. We introduce the following notation:

$$
\begin{array}{rlrl}
\mathbf{a} & =\left(a_{0}, \ldots, a_{2 m}\right) & i(\mathbf{a}) & =\left(b_{0}, \ldots, b_{2 m}\right) \\
\mathbf{a}^{b} & =\left(a_{0}^{\prime}, \ldots, a_{2 m}^{\prime}\right) & i(\mathbf{a})^{\lambda_{b}}=\left(b_{0}^{\prime}, \ldots, b_{2 m}^{\prime}\right) \\
i\left(\mathbf{a}^{b}\right) & =\left(c_{0}, \ldots, c_{2 m}\right) &
\end{array}
$$

Now, from the definition of "block," it follows that $a_{k-1}<a_{i}<a_{l+1}$ for all $i \in[k, l]$. From that observation, one can deduce that

$$
a_{i}^{\prime}= \begin{cases}a_{i} & \text { if } i \notin[k, l], \\ a_{i-1} & \text { if } i \in[k, l] \text { and } i \text { is even, } \\ a_{i+1} & \text { if } i \in[k, l] \text { and } i \text { is odd. }\end{cases}
$$

Indeed, it suffices to check that the sequence defined by this formula is valid $u$ symbol and that it satisfies the parity conditions in the definition of twisting. Both of these are easy to verify.

Next, it follows from the calculations in the proofs of parts (2) and (3) of Lemma 4.3 that

$\left\{b_{i} \mid i \in[k, l]\right.$ is odd and not isolated $\}=\left\{b_{i} \mid i \in[k, l]\right.$ is even and not isolated $\}$.

(This is proved there separately for staircases with even bottom and for ladders.) This observation lets us conclude that

$$
b_{i}^{\prime}= \begin{cases}b_{i} & \text { if } i \notin[k, l], \\ b_{i-1} & \text { if } i \in[k, l] \text { and } i \text { is even, } \\ b_{i+1} & \text { if } i \in[k, l] \text { and } i \text { is odd. }\end{cases}
$$


Finally, from the formula for $i$, we have

$$
b_{i}=\left\{\begin{array}{l}
a_{i}-i / 2 \\
a_{i}-(i+1) / 2
\end{array} \quad \text { and } \quad c_{i}= \begin{cases}a_{i}^{\prime}-i / 2 & \text { if } i \text { is even } \\
a_{i}^{\prime}-(i+1) / 2 & \text { if } i \text { is odd }\end{cases}\right.
$$

It follows that

$$
\begin{gathered}
c_{i}= \begin{cases}a_{i}-i / 2 & \text { if } i \notin[k, l] \text { and } i \text { is even, } \\
a_{i}-(i+1) / 2 & \text { if } i \notin[k, l] \text { and } i \text { is odd, } \\
a_{i-1}-i / 2 & \text { if } i \in[k, l] \text { and } i \text { is even, } \\
a_{i+1}-(i+1) / 2 & \text { if } i \in[k, l] \text { and } i \text { is odd }\end{cases} \\
=\left\{\begin{array}{ll}
b_{i} & \text { if } i \notin[k, l], \\
b_{i-1} & \text { if } i \in[k, l] \text { and } i \text { is even, } \\
b_{i+1} & \text { if } i \in[k, l] \text { and } i \text { is odd }
\end{array}=b_{i}^{\prime} .\right.
\end{gathered}
$$

This is the desired equality.

Proposition 4.10. The image of $\left.p\right|_{\bar{H}(\mathbf{a})}: \bar{H}(\mathbf{a}) \rightarrow \operatorname{Irr}(A(C))$ is precisely $\operatorname{Irr}(\bar{A}(C))$.

Proof. Recall that $\bar{A}(C)$ is defined to be the smallest quotient of $A(C)$ such that all local systems on $C$ that are assigned by the Springer correspondence to representations in the same two-sided cell as $\chi_{C, 1}$ actuall come from representations of $\bar{A}(C)$. Equivalently, $\operatorname{Irr}(\bar{A}(C))$ can be characterized as the smallest subgroup of $\operatorname{Irr}(A(C))$ that enjoys the following property:

For any $u$-symbol $\mathbf{a}^{\prime}$ that is similar to a, we have $\nu\left(\mathbf{a}^{\prime}\right) \in \operatorname{Irr}(\bar{A}(C))$

if and only if $i\left(\mathbf{a}^{\prime}\right)$ is congruent to $i(\mathbf{a})$.

Now, any $u$-symbol $\mathbf{a}^{\prime}$ similar to $\mathbf{a}$ arises by twisting $\mathbf{a}$ by a suitable set of ladders: $\mathbf{a}^{\prime}=\mathbf{a}^{\mu}$. If in fact $\mu \in \bar{H}(\mathbf{a})$, then the preceding proposition tells us that $i\left(\mathbf{a}^{\prime}\right)=$ $i(\mathbf{a})^{\lambda}$ for a suitable set of isolated points $\lambda$. Thus, $i\left(\mathbf{a}^{\prime}\right)$ and $i(\mathbf{a})$ are congruent, so $\nu\left(\mathbf{a}^{\prime}\right)=\nu\left(\mathbf{a}^{\mu}\right)=p(\mu)$ lies in $\operatorname{Irr}(\bar{A}(C))$.

Conversely, we see from Proposition 4.8 that the image of $\left.p\right|_{\bar{H}(\mathbf{a})}$ is an $\mathbb{F}_{2}$-vector space whose dimension is given by any of the following:

(number of blocks in $\mathbf{a})-1=$ (number of blocks with a bottom)

$$
=\text { (number of ladders with odd bottom) }
$$

It follows from the calculations in the proof of [2, Lemme 3.2] that the number of ladders with odd bottom is the same as the number of "even parts of odd height" (see [1] for the definition) in the partition labelling the unipotent class $C$. Finally, according to [1], the number of even parts of odd height is precisely the rank of $\bar{A}(C)$. Thus, the image of $\left.p\right|_{\bar{H}(\mathbf{a})}$ must be the full character group $\operatorname{Irr}(\bar{A}(C))$.

4.2. Type $B$. We let $\Psi_{2 n+1, m}$ (resp. $\left.\Phi_{2 n+1, m}\right)$ denote the set of sequences a $=$ $\left(a_{0}, a_{1}, \ldots, a_{2 m}\right)$ satisfying the following conditions:

$$
\begin{aligned}
& 0 \leq a_{0}, \quad 1 \leq a_{1}, \quad a_{i} \leq a_{i+2}-2, \quad \sum a_{i}=n+2 m^{2} \quad \text { for } \mathbf{a} \in \Psi_{2 n+1, m}, \\
& 0 \leq a_{0}, \quad 0 \leq a_{1}, \quad a_{i} \leq a_{i+2}-1, \quad \sum a_{i}=n+m^{2} \quad \text { for } \mathbf{a} \in \Phi_{2 n+1, m}
\end{aligned}
$$


The shift operations $S: \Psi_{2 n+1, m} \rightarrow \Psi_{2 n+1, m+1}, S: \Phi_{2 n+1, m} \rightarrow \Phi_{2 n+1, m+1}$ are given by

$$
\begin{array}{ll}
S(\mathbf{a})=\left(0,0, a_{0}+2, \ldots, a_{2 m}+2\right) & \text { for } \mathbf{a} \in \Psi_{2 n+1, m}, \\
S(\mathbf{a})=\left(0,0, a_{0}+1, \ldots, a_{2 m}+1\right) & \text { for } \mathbf{a} \in \Phi_{2 n+1, m} .
\end{array}
$$

As before, we put $\Psi_{2 n+1}=\lim _{m \rightarrow \infty} \Psi_{2 n+1, m}$ and $\Phi_{2 n+1}=\lim _{m \rightarrow \infty} \Phi_{2 n+1, m}$. These sets are again finite; we fix an $m$ large enough that $\Psi_{2 n+1} \simeq \Psi_{2 n+1, m}$ and $\Phi_{2 n+1} \simeq \Phi_{2 n+1, m}$. Elements of $\Phi_{2 n+1}$ (resp. $\Psi_{2 n+1}$ ) are called symbols (resp. $u$ symbols).

The bijection $i: \Psi_{2 n+1} \rightarrow \Phi_{2 n+1}$ is given by

$$
i\left(a_{0}, \ldots, a_{2 m}\right)=\left(a_{0}, a_{1}, a_{2}-1, a_{3}-1, \ldots, a_{2 m}-m\right) .
$$

Definition 4.11. A block is a subset $[k, l]$ of $[0,2 m]$ that meets one of the following conditions:

- $[k, l]$ is a union of consecutive parts $P_{1}, \ldots, P_{r}$, where $P_{1}$ and $P_{r}$ are ladders, $P_{1}$ is the unique part with even bottom, and $P_{r}$ is the unique part with odd top.

- $l=2 m$, and $[k, 2 m]$ is the union of consecutive parts $P_{1}, \ldots, P_{r-1}$, where $P_{1}$ is a ladder and the only part with even bottom, and where all parts have even top.

In either case, the bottom of the block is the bottom of $P_{1}$, and its top is the top of $P_{r}$, provided the latter is defined. That is, blocks meeting the second condition above are said to have no top.

Note that a part belongs to some block if and only if it is not a staircase with even bottom (and hence odd top).

Lemma 4.12. For any $\mathbf{a} \in \Psi_{2 n+1}$, there are an odd number of isolated points. Suppose $i(\mathbf{a})=\left(a_{0}^{\prime}, \ldots, a_{2 m}^{\prime}\right)$, and let $k_{0}, \ldots, k_{2 f}$ be the isolated points, numbered such that

$$
a_{k_{0}}^{\prime}<a_{k_{1}}^{\prime}<\cdots<a_{k_{2 f}}^{\prime} .
$$

If $\mathbf{a}$ is special, then $k_{t} \equiv t(\bmod 2)$.

As we did in type $C$, we will adhere to the convention introduced in this lemma for naming the isolated points. If $\mathbf{a}_{0}$ is a special symbol or $u$-symbol, we identify $V\left(\mathbf{a}_{0}\right)$ with the space $V_{\mathbf{c}}$ of Section 3.1 by

$$
e_{i}=\left\{k_{i}, k_{i+1}\right\}, \quad i=0, \ldots, 2 f-1 ; \quad e_{2 f}=\left\{k_{0}, k_{1}, \ldots, k_{2 f-1}\right\} .
$$

Lemma 4.13. Let $\mathbf{a}=\left(a_{0}, \ldots, a_{2 m}\right)$ be a distinguished $u$-symbol, and let $\mathbf{a}^{\prime}=$ $i(\mathbf{a})=\left(a_{0}^{\prime}, \ldots, a_{2 m}\right)$ be the corresponding symbol. Let $k$ and $l$ be two integers such that $0 \leq k<l \leq 2 m$.

(1) If $k$ and $l$ belong to distinct parts, then $a_{k}^{\prime} \neq a_{l}^{\prime}$.

(2) If $[k, l]$ is a staircase with odd bottom, then $k+1$ and $l-1$ are both isolated points. A staircase with even bottom contains no isolated points.

(3) If $[k, l]$ is a ladder, then $k$ is an isolated point if it is even, and $l$ is an isolated point if it is odd. There are no other isolated points in $[k, l]$.

(4) The number of isolated points in any part is congruent to the length of the part modulo 2.

(5) For each $t$, either $k_{t}<k_{t+1}$, or $k_{t}=k_{t+1}+1$ and $\left[k_{t+1}, k_{t}\right]$ is a staircase with odd bottom. In this case, $k_{t}$ and $k_{t+1}$ are both displaced. 
(6) Displaced isolated points occur in pairs $\left\{k_{t}, k_{t+1}\right\}=e_{t}$ with $t$ odd, with one such pair for each staircase with odd bottom.

4.3. Type $D$. Finally, let $\Psi_{2 n, m}$ (resp. $\left.\Phi_{2 n, m}\right)$ denote the set of sequences $\mathbf{a}=$ $\left(a_{0}, a_{1}, \ldots, a_{2 m+1}\right)$ satisfying the following conditions:

$$
\begin{aligned}
& 0 \leq a_{0}, \quad 1 \leq a_{1}, \quad a_{i} \leq a_{i+2}-2, \quad \sum a_{i}=n+2 m^{2}+2 m \quad \text { for } \mathbf{a} \in \Psi_{2 n, m}, \\
& 0 \leq a_{0}, \quad 0 \leq a_{1}, \quad a_{i} \leq a_{i+2}-1, \quad \sum a_{i}=n+m^{2}+m \quad \text { for } \mathbf{a} \in \Phi_{2 n, m}
\end{aligned}
$$

The shift operations $S: \Psi_{2 n, m} \rightarrow \Psi_{2 n, m+1}, S: \Phi_{2 n, m} \rightarrow \Phi_{2 n, m+1}$ given by

$$
\begin{array}{ll}
S(\mathbf{a})=\left(0,0, a_{0}+2, \ldots, a_{2 m+1}+2\right) & \text { for } \mathbf{a} \in \Psi_{2 n, m}, \\
S(\mathbf{a})=\left(0,0, a_{0}+1, \ldots, a_{2 m+1}+1\right) & \text { for } \mathbf{a} \in \Phi_{2 n, m} .
\end{array}
$$

Put $\Psi_{2 n}=\lim _{m \rightarrow \infty} \Psi_{2 n, m}$ and $\Phi_{2 n}=\lim _{m \rightarrow \infty} \Phi_{2 n, m}$. We fix an $m$ large enough that $\Psi_{2 n} \simeq \Psi_{2 n, m}$ and $\Phi_{2 n} \simeq \Phi_{2 n, m}$. Elements of $\Phi_{2 n}$ (resp. $\Psi_{2 n}$ ) are called symbols (resp. u-symbols).

The bijection $i: \Psi_{2 n} \rightarrow \Phi_{2 n}$ is given by

$$
i\left(a_{0}, \ldots, a_{2 m}\right)=\left(a_{0}, a_{1}, a_{2}-1, a_{3}-1, \ldots, a_{2 m}-m, a_{2 m+1}-m\right) .
$$

Definition 4.14. A subset $[k, l]$ of $[0,2 m]$ is called a block if it is a union of consecutive parts $P_{1}, \ldots, P_{r}$, where $P_{1}$ and $P_{r}$ are ladders, $P_{r}$ is the unique part with odd top, and $P_{1}$ is the unique part with even bottom. The top of the block is the top of $P_{r}$, and its bottom is the bottom of $P_{1}$.

A part belongs to some block if and only if it is not a staircase with even bottom (and hence odd top).

Lemma 4.15. For any $\mathbf{a} \in \Psi_{2 n}$, there are an even number of isolated points. Suppose $i(\mathbf{a})=\left(a_{0}^{\prime}, \ldots, a_{2 m+1}^{\prime}\right)$, and let $k_{0}, \ldots, k_{2 f+1}$ be the isolated points, numbered such that

$$
a_{k_{0}}^{\prime}<a_{k_{1}}^{\prime}<\cdots<a_{k_{2 f}}^{\prime} .
$$

If $\mathbf{a}$ is special, then $k_{t} \equiv t(\bmod 2)$.

If $\mathbf{a}_{0}$ is a special symbol or $u$-symbol, we identify $V\left(\mathbf{a}_{0}\right)$ with the space $V_{\mathbf{c}}$ of Section 3.1 by

$$
e_{i}=\left\{k_{i}, k_{i+1}\right\}, \quad i=0, \ldots, 2 f .
$$

Lemma 4.16. Let $\mathbf{a}=\left(a_{0}, \ldots, a_{2 m+1}\right)$ be a distinguished u-symbol, and let $\mathbf{a}^{\prime}=$ $i(\mathbf{a})=\left(a_{0}^{\prime}, \ldots, a_{2 m+1}\right)$ be the corresponding symbol. Let $k$ and $l$ be two integers such that $0 \leq k<l \leq 2 m+1$.

(1) If $k$ and $l$ belong to distinct parts, then $a_{k}^{\prime} \neq a_{l}^{\prime}$.

(2) If $[k, l]$ is a staircase with odd bottom, then $k+1$ and $l-1$ are both isolated points. A staircase with even bottom contains no isolated points.

(3) If $[k, l]$ is a ladder, then $k$ is an isolated point if it is even, and $l$ is an isolated point if it is odd. There are no other isolated points in $[k, l]$.

(4) The number of isolated points in any part is congruent to the length of the part modulo 2 .

(5) For each $t$, either $k_{t}<k_{t+1}$, or $k_{t}=k_{t+1}+1$ and $\left[k_{t+1}, k_{t}\right]$ is a staircase with odd bottom. In this case, $k_{t}$ and $k_{t+1}$ are both displaced.

(6) Displaced isolated points occur in pairs $\left\{k_{t}, k_{t+1}\right\}=e_{t}$ with $t$ odd, with one such pair for each staircase with even bottom. 
SPECIAL PIECES, SPRINGER CORRESPONDENCE, AND UNIPOTENT CHARACTERS 19

\section{REFERENCES}

[1] P. Achar, An order-reversing duality map for conjugacy classes in Lusztig's canonical quotient, Transform. Groups 8 (2003), 107-145.

[2] P. Achar and A.-M. Aubert, Supports unipotents de faisceaux caractères, to appear in J. Inst. Math. Jussieu.

[3] R. W. Carter, Finite groups of Lie type: conjugacy classes and complex characters, Pure and Applied Mathematics, John Wiley \& Sons, Inc., New York, 1985.

[4] H. Kraft and C. Procesi, A special decomposition of the unipotent variety, in Orbites unipotentes et représentations, III, Astérisque No. 173-174 (1989), 271-279.

[5] G. Lusztig, A class of irreducible representations of a Weyl group, Nederl. Akad. Wetensch. Indag. Math. 41 (1979), 323-335.

[6] G. Lusztig, Characters of reductive groups over a finite field, Ann. Math. Studies no. 107, Princeton Uninversity Press, 1984.

[7] G. Lusztig, Intersection cohomology complexes on a reductive group, Invent. Math. 75 (1984), $205-272$.

[8] G. Lusztig, Notes on unipotent classes, Asian J. Math. 1 (1997), 194-207.

[9] E. Sommers, Lusztig's canonical quotient and generalized duality, J. Algebra 243 (2001), 790-812.

[10] N. Spaltenstein, Classes unipotentes et sous-groupes de Borel, Lecture Notes in Math. no. 946, Springer-Verlag, Berlin-New York, 1982. 